\title{
Time, Fission, Fusion: An Argument against the Block Universe with Endurance*
}

\author{
Yuri Balashov \\ University of Georgia \\ Department of Philosophy \\ 107 Peabody Hall, Athens, Georgia 30602 \\ U.S.A. \\ yuri@uga.edu
}

Article info

CDD: 115

Received: 01.03.2017; Accepted: 14.03.2017

DOI: http://dx.doi.org/10.1590/0100-6045.2017.V40N1.YB

\author{
Keywords \\ Ontology of time \\ Endurance \\ Exdurance \\ Temporal experience
}

\begin{abstract}
Many philosophers believe in the Block Universe containing all objects and events - those that we intuitively call past, present, and future. But some of those who endorse this ontology of time also believe that objects persist by enduring - by being present in their entirety at all moments at which they exist. This combination of views, the Block Universe with Endurance, has survived the initial assault of the problem of temporary intrinsics and of several later objections. But I argue that the Block Universe with Endurance fails to account for a striking feature of our temporal experience and must be rejected in favor of the Block Universe with Exdurance.
\end{abstract}

*This paper revisits an older project (Balashov 2005). My thanks to Jordan Lavender, Mark Moyer, Laurie Paul, Brad Skow, and the members of UGA's Metaphysics Reading Group for many helpful discussions of the issues raised here. 


\section{Introduction}

Many philosophers believe in the Block Universe containing all objects and events - those that we intuitively call past, present, and future. According to this view, different times and their contents are like different places and their contents, all equally real; indeed times are simply certain regions of spacetime. But some of those who endorse this ontology of time and spacetime also believe that objects persist by enduring - by being present in their entirety (i.e. with no parts absent) at all moments at which they exist; or, in more recent terminology, by being multilocated at many instantaneous spacetime regions. This combination of views - call it the Block Universe with eNdurance (BUN) - has survived the initial assault of the problem of temporary intrinsics ${ }^{1}$ and of several later objections. ${ }^{2}$ But I argue below that BUN fails to account for a striking feature of our temporal experience and must be rejected in favor of the Block Universe with eXdurance (BUX). ${ }^{3}$

The argument is not entirely new. Versions of it have been developed and discussed, more or less favorably, by several authors. ${ }^{4}$ In this paper I am particularly concerned to close a glaring loophole in the argument. ${ }^{5}$ The key to this lies in drawing a tight analogy between exdurance and fission, and then using it to disable the "glaring objection." In \$1 I set up the initial problem for BUN. $\$ 2$ sketches a solution to it in the form of BUX, which crucially involves a parallel with the fission scenario. The scenario is detailed in $\$ 3$, and the parallel is further developed in $\$ 6$ to

${ }^{1}$ The problem that gave birth to the contemporary debate about persistence; see Lewis 1986: 202-204.

2 See, e.g., Merricks 1995.

3 'Exdurance' is a term often used, following Haslanger (2003), to describe persistence via stages. See $\$ 2$ below for details.

${ }^{4}$ See, in particular, Hoy 1978, Balashov 2005 and 2015, Torre 2010, Skow 2011 and 2015.

5 Another way to close the loophole was recently explored by Skow (2011). The connection between his way of closing the loophole and mine is discussed in $\$ 6$ below.

Manuscrito - Rev. Int. Fil. Campinas, v. 40, n. 1, pp. 109-143, jan.-mar. 2017. 
respond to a series of objections to the initial problem, discussed in $\iint 4$ and 5.

\section{The Initial Problem}

Tuesday was my last day on the skiing slopes in a beautiful part of the country with tons of snow, dry air, and out of this world mountains. It was cold and sunny, and I felt extremely elevated. The next day I had to rush back to that warm and wet place, which greeted me with the usual splash of swampy air, and I immediately felt very depressed. Figures $1 \mathrm{a}$ and $1 \mathrm{~b}$ represent my feelings in the Block Universe: elevated on Tuesday, depressed on Wednesday. Think of them as mental episodes tied up to their locations in spacetime, much like concerts or breakfasts.

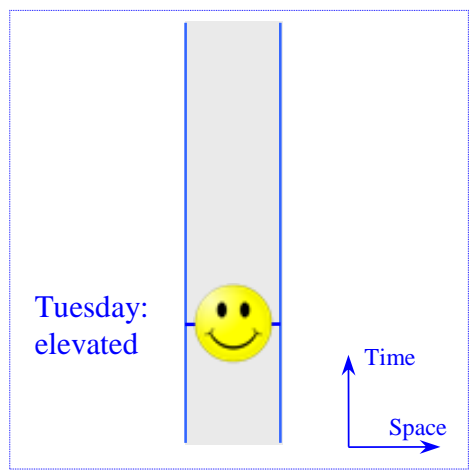

(a)

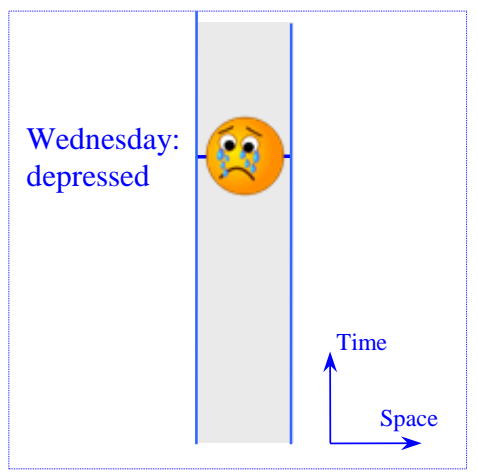

(b)

Fig. 1: My feelings in the Block. Universe: elevated on Tuesday, depressed on Wednesday. The shaded rectangle represents my path in spacetime.

But the episodes in question are my experiences. Despite the drastic difference in their phenomenal character they are ontologically on par and have equal claims to represent my perspective on the Block Universe; after all, there is only one me in the Block Universe, but many times and many experiences. I believe, however, that I am viewing the Block Universe exclusively from the Wednesday perspective (tainted with depression), and 
not from the Tuesday perspective. This belief may or may not be true, but I have it, and it needs to be explained. However, the Block Universe with eNdurance (BUN) lacks the resources to do so because it connects me with my elevation (and with Tuesday) as well as with my depression (and Wednesday) by equally solid arrows (see Fig. 2 below):

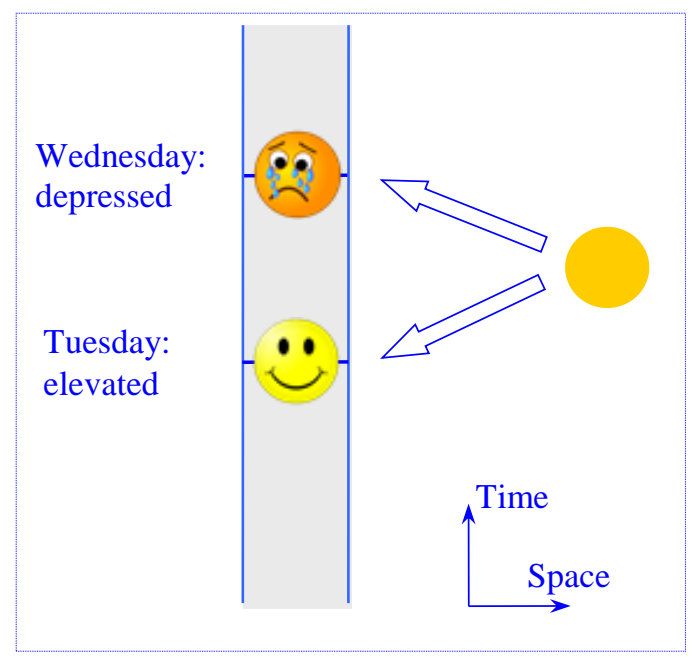

Fig. 2: My feelings in the Block Universe with eNdurance (BUN). The circle on the right represents my enduring self, the upper arrow the relations wholly present at and depressed at, the lower arrow the relations wholly present at and elevated at.

A gut reaction to this opening salvo is to dismiss it as based on an obvious confusion (e.g., between the tensed and tenseless uses of verbs such as 'view' and 'feel') or neglect (e.g., of the need for a semantic reduction of tense, of the lessons of the essential indexical, etc.). Here is one way to put the dismissal on behalf of the BUNist: I am elevated and view the Block Universe from the Tuesday perspective, on Tuesday; and I am depressed and view the Block Universe from the Wednesday perspective, on Wednesday; and that's all there is to it. I have these perspectives one at a time, not simultaneously. Since today is Wednesday

Manuscrito - Rev. Int. Fil. Campinas, v. 40, n. 1, pp. 109-143, jan.-mar. 2017. 
my belief in the exclusive nature of my Wednesday perspective is thereby explained.

But it is not. The gut reaction does show there is something imprecise in the opening salvo. However, the gut reaction leaves a really pressing explanatory question unaddressed. The imprecision of the opening salvo has to do with a potentially misleading aspect of spacetime diagrams such as Fig. 2, where both arrows are drawn at once. This may suggest that the challenge for the BUNist is to explain (i) why I never have my experiences from different times together. But this is easy to handle (see below). The real challenge is to explain (ii) why I have one exclusive temporal experience (e.g. my Wednesday experience) rather than another (e.g. my Tuesday experience)? More precisely: why do I believe that I have exclusively the former rather than exclusively the latter?

To elaborate, Fig. 2 represents the "view from nowhere" true to the spirit of the BUN ontology. Among other things, it is supposed to provide a metaphysical explanation of my temporal experiences. In particular, the arrow connecting me to Tuesday and elevation explains why I feel elevated on Tuesday and not also on Wednesday, and why I do not feel depressed on Tuesday; and similarly for the arrow connecting me to Wednesday and depression. In short, the arrows do explain why I can only have my temporal experiences one at a time and not together: there is simply no arrow connecting me to both. This helps with (i), but not with (ii); for nothing in Fig. 2 privileges the arrow connecting me to Wednesday over the arrow connecting me to Tuesday; and therefore, nothing in Fig. 2 explains why I have one exclusive temporal experience (my Wednesday experience) rather than another (my Tuesday experience).

So what is wrong with the initial gut reaction? Specifically, what is wrong with its bottom line: "Since today is Wednesday, my belief in the exclusive nature of my Wednesday perspective is thereby explained?" What is wrong with it is an illegitimate appeal it makes to the alleged fact that "today is Wednesday," which has no foundation in the BUN ontology and, therefore, cannot be used to underwrite my wrong belief in the exclusive presence of my Wednesday perspective. The Tuesday perspective is equally mine, and nothing in the Block Universe with endurance makes it the case that I should (believe that I) have my Wednesday perspective rather than my Tuesday perspective. The real 
question then is the "rather than" question, not the "why not all at once" question.

This is, hopefully, an improvement on the opening salvo. To be sure, there is a long way to go. The committed friends of BUN and the skeptics are, no doubt, ready to press on with further objections. I will deal with them in $\$ \int 4$ and 5 . At this point I want to jump forward and consider a rather radical dissolution of the puzzle about elevation and depression assuming for now that it is a real puzzle. ${ }^{6}$

\section{The Block Universe with Exdurance}

Why do I feel depressed and find myself on Wednesday rather than elevated and finding myself on Tuesday, despite the fact that my elevation and the time at which it is felt are every bit as real as my depression and the corresponding time? The really radical way to resolve - or, rather, dissolve - this puzzle for the Block Universe theorist is to abandon one of its presuppositions, the idea that said experiences and many others belong to a single subject, and attribute them instead to numerically distinct stages of me. My Wednesday stage is depressed, finds itself only on Wednesday (more precisely, at a certain moment on Wednesday) and in Georgia, thus giving me a false belief about my exclusive presence on Wednesday and about my exclusive depression. My Tuesday stage is elevated, finds itself only on Tuesday and in Colorado, thus giving me another false belief that I am exclusively present on Tuesday and feel exclusively elevated (Fig. 3). Importantly, in having the first belief and the

\footnotetext{
${ }^{6}$ As stated here, the puzzle has something in common with Arthur Prior's famous "thank Goodness that's over" puzzle, but is more general in scope, as it pertains to a whole variety of present temporal experiences. They include, but are not restricted to, differential attitudes towards the past and the future, such as relief, anticipation, or regret. The parallels as well as the differences between a version of the present puzzle and Prior's problem are explored in Skow 2011 (see, in particular, note 1) and 2015. In my discussion I will focus exclusively on the puzzle about present experiences.
}

Manuscrito - Rev. Int. Fil. Campinas, v. 40, n. 1, pp. 109-143, jan.-mar. 2017. 
corresponding experiences, I am not aware of having the second belief, along with the corresponding experiences.

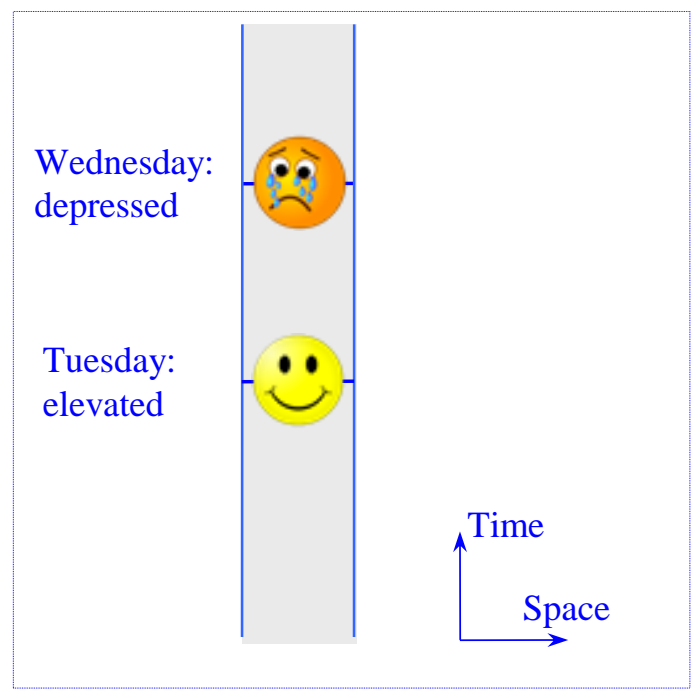

Fig. 3: My feelings in the Block. Universe with eXdurance (BUX).

In familiar terms, this move amounts to rejecting endurance in favor of exdurance, or "persistence by stages." The Block Universe with eXdurance (BUX) therefore seems to be an apt name for the resulting combination of views about time and persistence, and I shall use it in the sequel. The ontology of exdurance, also known as stage theory, is usually characterized as a temporal counterpart theory. ${ }^{7}$ I hasten to note that while I appreciate the exceedingly popular point of drawing analogies between persistence across time in BUX and "persistence across worlds" in the modal counterpart theory I am not enthusiastic about pursuing such parallels too far right from the start. My thinking about BUX is guided instead by the

${ }^{7}$ For exposition and defense of stage theory, see Sider 2001 and Hawley 2001. As far as I know, the term 'exdurance' was first used to describe persistence via stages by Haslanger (2003).

Manuscrito - Rev. Int. Fil. Campinas, v. 40, n. 1, pp. 109-143, jan.-mar. 2017. 
"not aware" aspect of the above description of my temporal experiences and of the corresponding beliefs about their exclusive nature.

Let me elaborate on the difference between these two approaches to developing the ontology of exdurance. The usual approach starts by focusing on the indexical nature of actuality in certain theories of modality, adds the counterpart analysis of de re modal predication, formulates their precise temporal analogs, and then lets the chips fall where they may. When applied to me and my temporal experiences, the approach yields the verdict that, strictly speaking, I exist only on Wednesday ${ }^{8}$ and am depressed simpliciter, while someone else - my temporal counterpart - exists on Tuesday and is elevated simpliciter. This explains the sense in which, while I am simply depressed, I was elevated, courtesy my Tuesday counterpart. It also accounts for the derivative sense in which I exist on Tuesday (and Monday, etc.), similar to the sense in which Hubert Humphrey exists according to many worlds in the ontology of modal realism, even though, strictly speaking, he exists only at one world. ${ }^{?}$ Pursuing the modal-temporal analogy further should lead me to conclude that, in the most fundamental way, I exist only in the present. And if I exist at another time this is only in a less fundamental way reflected in our everyday use of non-present tenses and other linguistic devices. Accordingly, those sympathetic to this approach should redraw Fig. 3 as follows (Fig. 4):

\footnotetext{
${ }^{8}$ That is to say, at a certain moment on Wednesday.

${ }^{9}$ On the difference between existing at a world and according to a world in modal realism, see Lewis 1986: 96, especially note 61. For a recent discussion of the temporal analogs of these in BUX, see Skow 2015: \$12.3.
}

Manuscrito - Rev. Int. Fil. Campinas, v. 40, n. 1, pp. 109-143, jan.-mar. 2017. 


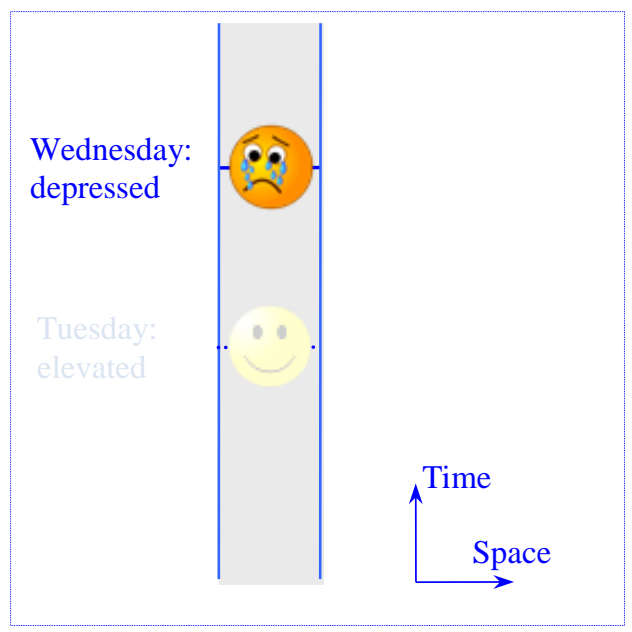

Fig. 4: The solid face represents me. The washed-out face represents someone else. ${ }^{10}$

Although I see the ideological pressures pushing one in this direction the outcome strikes me as inadequate. While it may be of great help in developing a formally elegant account of temporal predication in BUX it fails to provide for a good ontological starting point. And in the context of this particular problem, pursuing a decidedly linguistic task before securing a plausible ontological foundation seems to me to be putting the cart before the horse. Consider Fig. 3 versus Fig. 4 again. These figures seek to depict the ontology of BUX, and both are drawn "from nowhere," not from any vantage point in time. (Contrast them with Figs. 1a and 1b, which are drawn from two different vantage points in time.) In this atemporal perspective there is no reason to draw one face as solid and the other as washed out. This is where the modal-temporal analogy breaks down: supposing there are both temporal and modal counterparts, our attitude towards the former is different from our attitude towards the latter.

${ }^{10}$ Fig. 4 follows the solid-dashed style of Skow's Fig. 9 (2011: 381).

Manuscrito - Rev. Int. Fil. Campinas, v. 40, n. 1, pp. 109-143, jan.-mar. 2017. 
To make the contrast vivid, suppose you hand me all my temporal stages from different times, including the present. Putting myself squarely in the shoes of the BUXist, there is a transparent sense in which I am all of them - "severally but not jointly," as the saying goes. But if I am given all my modal counterparts from different worlds I will hesitate to say the same. No, in this case the right thing to say is that I am this guy (who does philosophy), and that guy (who went into politics) is someone else indeed. To my mind, this shows that the modal-temporal analogy has its limits and should be used with care. Our attitude towards out temporal counterparts is decidedly different from our attitude towards our modal counterparts.

I may be led to this conclusion because, while I am a realist about times, I am not a modal realist. I believe times and their contents are simply there, and the present time is not special, whereas possible worlds are not there, and the actual world is very special. But I also think this differential attitude towards times and worlds is reasonable. This is not to say that the modal-temporal analogies are useless. Their role in temporal predication must eventually be explored. But we should be in no rush to take the ideology and conceptual structure of modal realism as a blueprint for the ideology and conceptual structure of the theory of persistence.

Here is an alternative starting point suggested by further reflection on the puzzle of temporal experience. Begin by thinking of self-locating beliefs ${ }^{11}$ and of what determines a subjective perspective and a corresponding attitude de se in the Block Universe. ${ }^{12}$ Generally speaking, there are three dimensions of self-location at work here: modal, personal, and temporal. To explain actual experiences one needs, for starters, to know one's location in the space of possible worlds. But that is not enough; one also needs to know one's location within a world; and for the latter, one needs to know (i) who

\footnotetext{
${ }^{11}$ See Perry 1979.
}

${ }^{12}$ See Lewis 1979 and a useful discussion in Skow 2015, Appendix to Ch. 12.

Manuscrito - Rev. Int. Fil. Campinas, v. 40, n. 1, pp. 109-143, jan.-mar. 2017. 
one is and (ii) when it is. ${ }^{13}$ In discussions of self-locating beliefs, the modal dimension often, and for a good reason, remains in the background. But it takes center stage in many accounts of exdurance where it is used not only as a good model for (ii), but as a key to developing a general account of BUX. I suggest that in drawing metaphysical lessons from temporal experience we should start by aligning (ii), the temporal dimension of the situation, with the equally central and prominent personal dimension (i), and not with the background modal dimension. We should take a metaphysically appropriate answer to the who I am question as a model for answering the when it is question; and then let the chips fall where they may. They may eventually land in the same ballpark, but in a somewhat different pattern. But we should start from the right place, by giving priority to the most important and salient questions.

BUX is uniquely positioned to handle (i) - the "who one is" dimension of self-location in the Block Universe, and (ii) - the "when it is" dimension of it, in a uniform way because it can treat them as two aspects of a single question about the self-location of person-stages in the Block Universe. Why do I find myself on Wednesday and depressed? Because I am my Wednesday stage, which cannot find itself anywhere else; in particular, I am neither your Wednesday stage nor my Tuesday stage. BUN, on the other hand, approaches the personal and temporal dimensions of selflocation rather differently, by disallowing entities that "cannot find themselves anywhere else" in time. ${ }^{14}$ It individuates selves in a more fine-grained way than it does times. It responds to the who I am question by pointing to a long spacetime path (the shaded region in

${ }^{13}$ Doesn't one also need to know where one is? As Perry notes, "it is plausible to suppose that other indexicals [including spatial indexicals] can be eliminated in favor of 'I' and 'now"' (1979: 16).

${ }^{14}$ With the possible exception of instantaneous entities that exist no longer than a moment.

Manuscrito - Rev. Int. Fil. Campinas, v. 40, n. 1, pp. 109-143, jan.-mar. 2017. 
the above figures) stretching from my birth to death. This exhausts the resources of the theory prior to addressing the when it is question, thus precluding it from explaining my belief in the exclusive presence of Wednesday and depression.

Of course, things are not so simple. By embracing entities that "cannot find themselves anywhere else" in time, BUX puts on the table too many of them. I find myself on Wednesday, depressed, and believing in the exclusive presence of Wednesday, because I am my Wednesday stage. But I also find myself on Tuesday, elevated, and believing in the exclusive presence of Tuesday, because I am also my Tuesday stage. This is resisted by advocates of exdurance who are carried away by the modal counterpart theory. And this is exactly where I am inclined to part ways with them. It just seems non-negotiable to me that my Tuesday stage has an equal claim to represent my perspective, and not the perspective of someone else, in the Block Universe with eXdurance.

But to say this is not to say that I persist by enduring. We need to walk a fine line here. Endurance implies strict multilocation (represented in Fig. 2 above) and, as noted above, bans items confined to single times. Exdurance of all types embraces such items and disallows strict multilocation. Exdurance of the type I favor goes beyond this claim and allows for a sense in which all my temporal stages are on par and equally eligible to represent my perspective on the Block Universe - severally but not jointly. Although the relation between me and my stages is not identity, ${ }^{15}$ it incorporates "everything that matters." And what matters in this case goes along with what allows me to say that I have my Wednesday perspective, as well as my Tuesday perspective.

What needs to be added, however, is the claim that in having my Wednesday perspective, I am not aware of having my Tuesday

15 What is it? This is a difficult question that cannot be addressed here. But see note 32 for some options.

Manuscrito - Rev. Int. Fil. Campinas, v. 40, n. 1, pp. 109-143, jan.-mar. 2017. 
perspective. The "not aware" part comes from tightening the analogy between the personal and temporal aspects of self-location (while keeping the modal aspect at safe distance for now).

\section{Fission and Stages of the Self}

Recall the lessons some philosophers have drawn from fission, a scenario in which I am physically and/or psychologically continuous with two future persons, Lefty and Righty. Suppose that after the fission, Lefty remains on the mountain trails and elevated, and Righty ends up in the swampy place, severely depressed (Fig. 5).

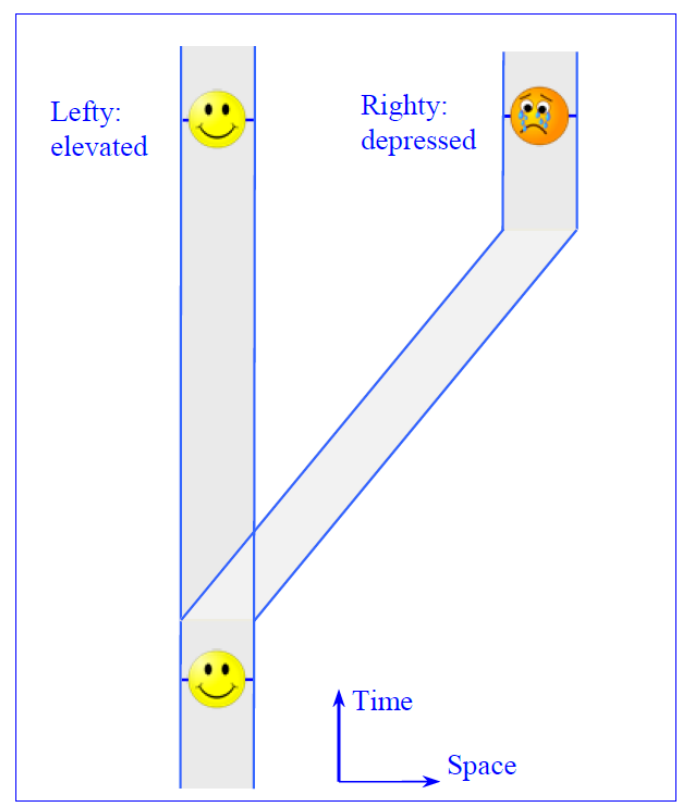

Fig. 5: Fission.

Manuscrito - Rev. Int. Fil. Campinas, v. 40, n. 1, pp. 109-143, jan.-mar. 2017. 
Different philosophers have said different things about the relationship between me, Lefty, and Righty in such scenarios. ${ }^{16}$ According to the most pessimistic verdict favored by many endurantists, I cease to exist and two new persons are created. On a more sanguine but rather implausible view, there are two coincident persons right from the start whose trajectories and experiences diverge later. Another approach starts by saying that there is only one pre-fission person, me, who survives both as Lefty and as Righty without, however, being strictly identical to either of them; and then lets the chips fall where they may. This yields the verdict that, strictly speaking, I do not exist post-fission, although there is a sense in which I will be Lefty, and I will be Righty.

To me, this still falls short of the expectations usually stated in discussing fission scenarios. They typically involve the psychological continuity considerations. If I am moved by such considerations at all I should not settle for anything else than full-blown existence after fission that is every bit as good - or bad, as the case may be - as my pre-fission existence. The apersonal perspective of Fig. 5 makes it clear that my prefission belief that I am me, Lefty's belief that he is me, and Righty's belief to the same effect all have exactly the same metaphysical force and must therefore be treated equitably. Moreover, Lefty's and Righty's beliefs that they are me are equally well described as my beliefs that I am Lefty and that I am Righty. Of course, we should add an all-important point: in believing that I am exclusively Righty, depressed, and in the swampy place, I am not aware of Lefty's contrary beliefs. We should start with these indubitable observations and see where they lead us.

In a way, they lead us to the picture of the "fragmented" or "scattered" self sketched by Parfit and others. ${ }^{17}$ It is forced upon us by a dilemma arising for anyone who takes the psychological criterion of personal persistence seriously. If either branch of the forking path in Fig. 5 is me then both of them are - "severally" but not jointly. The only reasonable

16 See, in particular, Parfit 1971, Lewis 1983, and Sider 2001: 201-204. The options mentioned in text do not exhaust all the possibilities discussed, developed and defended by many philosophers who have written on fission.

${ }^{17}$ Parfit refers to it as the "bundle theory" of the person. See his 2008.

Manuscrito - Rev. Int. Fil. Campinas, v. 40, n. 1, pp. 109-143, jan.-mar. 2017. 
alternative is to say that neither of them is me. Much of Parfit's work can be read as denying a substantive difference between these two alternatives. I am less interested in taking a stance on this issue than in tightening the analogy with the temporal case. The analogy should by now be clear. In fact, the self that is scattered across the personal dimension of Fig. 5 is also scattered across the temporal dimension of it. ${ }^{18}$ And the explanation of Righty's illusion that he alone is me (or my illusion that I only have the experiences occurring at a single person, Righty) is exactly parallel to the explanation of my illusory belief that I only have the experiences occurring in the unique present. In both cases, there is no way around some sort of disunity and compartmentalization in the structure of the self. ${ }^{19}$

\section{Further Objections and Replies}

But there is also no doubt that these consequences raise the cost of BUX. This should inspire BUNists to press their original skepticism about the puzzle of temporal experience. If there is no puzzle in the first place there is no need to spend so much effort dissolving it. Let us revisit the puzzle - alleged or real, as the case may be - and the emerging rival positions.

The Puzzle. Why do I believe that I am exclusively present on Wednesday and exclusively depressed, rather than being exclusively present on Tuesday and exclusively elevated, despite the fact that

\footnotetext{
18 And as a consequence, in the spatial dimension as well. Again see Perry (1979: 16) on eliminating the spatial dimension of self-location in favor of the personal and temporal dimensions.

19 See Bayne 2010, who describes and evaluates various forms of "static disunity" (i.e. disunity at a time) that may be manifested in unusual experiences, including the "split-brain syndrome," such as representational, access, and phenomenal disunity. It should be noted that Bayne's analysis is firmly grounded in real-life clinical cases, does not extend to gedanken experiments or to personal persistence across time, and is intended to support the phenomenal unity thesis.
}

Manuscrito - Rev. Int. Fil. Campinas, v. 40, n. 1, pp. 109-143, jan.-mar. 2017. 
both times and experiences are equally real, and my relations to both have the same ontological standing in the Block Universe?

The BUXist takes the Puzzle seriously and insists that, to deal with it properly, the structure of the self must, in effect, be made as fine-grained as the structure of time. It must be as fine-grained as is needed to establish precise self-location of the subjects of experience in the Block Universe. BUN falls short of this requirement. It partitions the entire experiencebearing content of the Block universe into distinct personal bundles individuated by long spacetime trajectories representing their life careers. But it stops there. BUX, on the other hand, takes the next logical step in pinpointing self-location. By attributing different temporal experiences to distinct bearers, BUX establishes parity among times and selves thereby eliminating the nagging "rather than" question. But it must pay a cost, by embracing the compartmentalized self.

The BUNist, in contrast, sees no need in the compartmentalized self or, for that matter, in replacing endurance with exdurance. And she thinks that the Puzzle is based on a confusion. The confusion does not consist in mistakenly attributing to me a mix of elevation and depression (see $\$ 1$ ). Rather it lies in using a misleading and tendentious language to create a false impression that the whole story about my temporal experiences involves more than is represented in Fig. 2, in which a single subject is connected to multiple times and multiple experiences. Locating the confusion should make it clear that there is nothing more to the story. This can be done in a number of related ways. The BUXist, however, will argue that the Puzzle emerges from all such objections unscathed.

Let me consider several representative objections on behalf of the BUNist, along with the corresponding replies on behalf of the BUXist. ${ }^{20}$

Objection 1. I am elevated on Tuesday, and I (the selfsame subject) am depressed on Wednesday, and that is all. Portraying the need to explain away my illusory belief that I am exclusively present on Wednesday and exclusively depressed (rather than being exclusively present on Tuesday and exclusively elevated) as involving a serious problem or difficulty turns

${ }^{20}$ For some earlier versions, see Balashov 2005. I am grateful to many people who have spelled out or suggested these or similar objections.

Manuscrito - Rev. Int. Fil. Campinas, v. 40, n. 1, pp. 109-143, jan.-mar. 2017. 
on overlooking the obvious connection between Wednesday and depression, and generates gratuitous questions: Why do I find myself on Wednesday rather than Tuesday? and Why am I depressed rather than elevated? Recalling that Wednesday is precisely when I am depressed takes the mystery out of both questions.

Reply 1. But it doesn't. There is a similar connection between Tuesday and my elevation: Tuesday is exactly when I am elevated. What I want to know is why I am finding myself on Wednesday and depressed, rather than on Tuesday and elevated. Nothing in my relations to these dates and corresponding experiences favors one pair over the other. I do believe, however, that it is Wednesday and I am depressed. I do not believe that it is Tuesday and I am elevated. The Block Universe theorist of any stripe must categorize such exclusive beliefs as illusory. But if she is an endurantist she must locate the origin of the illusion in Fig. 2. And nothing in this figure is capable of connecting me to Wednesday and depression, without also connecting me to Tuesday and elevation.

Objection 2. I am depressed, find myself on Wednesday, and believe (wrongly) in the exclusive presence of Wednesday and depression, because my believing it occurs on Wednesday. I am elevated, find myself on Tuesday, and believe (wrongly) in the exclusive presence of Tuesday and elevation, because my believing it occurs on Tuesday. All believing, wondering, contemplating and so forth is done at times, and many such mental acts are correlated with corresponding experiences. Any further belief in the exclusive presence of one such experience correlated with a particular act of wondering about it, as opposed to another, is illusory because the correlation (fully reflected in Fig. 2) remains "external" to it. ${ }^{21}$

Reply 2. External or not, the correlation in question must explain my illusory belief in the exclusive occurrence of a particular set of correlated items, as opposed to another such set. The alleged explanation is supposed

${ }^{21}$ Earlier versions of the "correlation analysis" of the experience of the present by B-theorists of time can be found in Grünbaum 1976, Oaklander 1994, and Mellor 1998: Ch. 4. In his very balanced evaluation of Grünbaum's minddependence view of becoming, Ronald Hoy (1978) sketched a view that is very close to my own (i.e. a version of BUX). For a more recent and particularly focused presentation of the "correlation objection," see Skow 2011: 376.

Manuscrito - Rev. Int. Fil. Campinas, v. 40, n. 1, pp. 109-143, jan.-mar. 2017. 
to go as follows: I believe (wrongly) in the exclusive occurrence of my depression because it occurs on Wednesday, and Wednesday is the day when I am contemplating it. But by the same token, I also believe (wrongly) in the exclusive occurrence of my elevation because it occurs on Tuesday, and Tuesday, let us suppose, is the day when I am contemplating it. What I want to know, however, is why I am depressed and contemplating my depression, rather than elevated and contemplating my elevation. Nothing so far explains the seeming occurrence of the first correlated pair, over the second.

Objection 3. But the first correlated pair occurs on Wednesday, and today is Wednesday.

Reply 3. In light of Replies 1 and 2, the BUNist should not expect any help from adding Wednesday to the first set of correlated items, for Tuesday could then be added to the second such set. This will make both sets bigger, while still leaving us without an explanation of why the first, and not the second set, seems to be occurring. What about "today is Wednesday"? Read one way it is simply false: nothing in the Block Universe (with eNdurance or eXdurance) makes it the case that today is Wednesday. A more charitable reading of "today is Wednesday" would interpret it as someone's token utterance or thought happening on Wednesday. But it should be clear by now that nothing that happens on Wednesday (including anyone's token utterances and thoughts), in addition to my experiences and my contemplation of them, will help with our problem, for corresponding happenings of the same sort can always be presumed to occur on Tuesday as well, in addition to my Tuesday experiences.

Think of the correlations invoked in Objection 2 as $n$-tuples involving me, times, my temporal experiences, my acts of contemplating them, and any number of other events happening at different times, such as various token utterances or thoughts, other people's reports of my condition, calendars showing particular dates, and so forth. Objection 2 attempts to locate the source of my illusory belief in the exclusive occurrence of my depression in the triple <me, my depression, my contemplation of my depression>, whereas Objection 3 invokes more complicated $n$-tuples such as <me, Wednesday, my depression, my contemplation of my depression $>$ or $<$ me, Wednesday, my depression, my contemplation of 
my depression, Ted's observation that I am depressed, Fred's token thought that today is Wednesday, Trump's election victory, ...>. The replies to both objections bring to the table the corresponding $n$-tuples involving me, Tuesday, my elevation, my contemplation of my elevation, and/or any number of things happening on Tuesday, and point out the recurrence of the selfsame subject in both $n$-tuples, namely me. This puts me en rapport with the Tuesday, as well as the Wednesday items, in full accordance with the ontology of BUN. The presence of the same me in all such $n$-tuples, however, stands in the way of explaining why the parity between them appears to be broken on the way to phenomenology. In the absence of the fragmented self, my temporal experiences are not sufficiently compartmentalized to generate the relevant illusory beliefs.

Objection 4. Both the alleged Puzzle and the replies to the above objections put a lot of weight on the "rather than" question: Why do I believe that I am exclusively depressed and exclusively en rapport with various Wednesday items, rather than being exclusively elevated and en rapport with the corresponding Tuesday items? - and then directs the reader to Fig. 2 for an answer. But Fig. 2 depicts an external (tenseless, atemporal, God's eye) perspective where I am en rapport with both, and the "rather than" question makes no sense in it. It makes sense only from the internal temporal perspectives of Figs. $1 \mathrm{a}$ and $1 \mathrm{~b}$, where the answer in each case is obvious.

Reply 4. This is incorrect. The "rather than" question is not a question asked from a particular internal perspective. It is a question about the obtaining of a certain internal perspective, rather than another such perspective. And questions of this sort can be posed meaningfully only in an external perspective. To be sure, there is an important connection between the two types of perspective: the external ontological perspective of Fig. 2 is supposed to explain why I view the Block Universe from a particular internal perspective, that of Fig. 1b, rather than another such perspective. But it cannot; for it gives us only one viewer but many internal perspectives. For genuine explanation, each internal perspective must be associated with a separate viewer. And this calls for a radical revision of the "I" part of the metaphysical equation, not just the temporal part.

Manuscrito - Rev. Int. Fil. Campinas, v. 40, n. 1, pp. 109-143, jan.-mar. 2017. 
The BUNist will not stop here. She will say: I do view the Block Universe from many internal perspectives, just at different times. This will bring us back to Objection 1 . Have we made any progress?

\section{The Stalemate}

Sometimes I think that we have and that BUX has an upper hand. But at other times it seems to me that the only net result of this and similar exchanges is the clarification and consolidation of the rival positions. Perhaps we have reached a point where one party simply refuses to see a problem which the other deems real and important. The stalemate can be traced back to the central ontological disagreement between BUN and BUX. The latter posits entities - person stages - that (i) have temporal experiences, beliefs, and so forth simpliciter; (ii) are located at single times; and (iii) cannot find themselves anywhere else or with other experiences and beliefs. The BUXist then argues that, since BUN denies the existence of such entities it cannot adequately explain: (i) the exclusive occurrence of particular temporal experiences and accompanying beliefs; (ii) the peculiar sense, foreign to the ontology of the Block Universe, in which the subject finds itself at a particular time rather than a different time; (iii) and other, "thicker" combinations of illusory beliefs. The BUNist, however, insists that all such data can be explained in the manner of Fig. 2, without compartmentalizing the self into separate stages that are unaware of each other's existence and experiences. We just need to remember that experiences are had at times. There is no such thing as being depressed simpliciter, but only depressed at $t$. And there is no such thing as occurrence simpliciter, only occurrence at t. Any belief in the exclusive occurrence of particular temporal experiences is illusory, not because of the subject's unawareness of earlier and later numerically distinct subjects of similar experiences, but because of the very natural disregard of the relational nature of the experiences and other mental states involved in the situation. In some circumstances (such as those of the present discussion), this disregard gives rise to a confusion or conflation of tensed and tenseless locutions, first- and third-person perspectives, to the neglect of the

Manuscrito - Rev. Int. Fil. Campinas, v. 40, n. 1, pp. 109-143, jan.-mar. 2017. 
distinctive behavior of indexicals, of the need for a semantic reduction of tense, and other related oversights.

Let us summarize the BUNist stance as follows: All temporal experiences and accompanying beliefs are at bottom relational. This stance uniformly underlies the initial skeptical reaction to the Puzzle and all the subsequent objections. The BUXist replies also have a unifying leitmotif: a consistent denial of the relational nature of temporal experiences and attendant beliefs. The disagreement is rather theoretical. Neither party denies that I am elevated on Tuesday and depressed on Wednesday. But one party insists that this is enough to explain everything that needs to be explained. And the other considers it as a halfway house on the road to the compartmentalized self.

All things being equal, it would be good to have an independent argument against the view that all temporal experiences are at bottom relational. I develop it below. ${ }^{22}$

\section{More on Times and Persons}

Recall the temporal-personal analogy used in $\$ 3$ to sketch an alternative approach to the ontology of BUX. Different times were likened to numerically distinct persons there, rather than to different worlds. The fission scenario was then invoked to drive home the central point of the analogy. Righty is depressed and finds himself exclusively in the swampy place. He also thinks he is uniquely me. In this he is wrong, for Lefty, who is elevated and finds himself exclusively in the mountains, has an equal claim to be me (see Fig. 5). In these respects, Lefty and Righty are a lot like me-on-Tuesday and me-on-Wednesday from the original puzzle. Both pairs are segments of the compartmentalized self that is scattered, respectively, across the personal and the temporal dimensions. Each member of a pair (i.e. Righty versus Lefty, and me-on-Wednesday versus

22 The argument bears resemblance to another gedanken experiment to the same effect in Skow 2011: 376-379. Skow makes no use of fission but explores a spatial analogy involving a hypothetical agent simultaneously having different experiences at different places.

Manuscrito - Rev. Int. Fil. Campinas, v. 40, n. 1, pp. 109-143, jan.-mar. 2017. 
me-on-Tuesday) is unaware (let us assume) of the existence of the other member. Relatedly, in having a given experience, each member is not aware of the corresponding experience of the other member. This is what the BUXist should say, anyway.

Consider now a hypothetical BUNist who is attracted to the temporalpersonal analogy. ${ }^{23}$ She may, for example, be moved by the alleged success of the relativization strategy in the temporal case and consider it a good model for resolving the older problems with fission, which present a prima facie challenge for strict identity over time. What will she say? If she embraces the analogy she must deny the need for a compartmentalized self in either case. In particular, she must insist that Lefty and Righty are not separate subjects of experience but a single subject, me, having different experiences at different persons. In other words, she must insist that my personal experiences are "at bottom relational": I am elevated at Lefty and depressed at Righty. She will note that I am not inclined to describe my experiences this way simply because I am not aware of their relational nature. When apprised of the situation I should agree that, in addition to being depressed, I am also elevated, just at different personal indices. My exclusive belief that I am depressed simpliciter is thus illusory. Experiences do not occur simpliciter, but only at persons. As an added consequence, I am in the mountains at Lefty, and in the swampy place at Righty.

To summarize this hypothetical theoretical development in a slightly different terminology, the BUNist begins with the core endurantist ideas of multilocation in time ("persisting objects are wholly present at all moments at which they exist") and of relativization of experiences to times (experiences are, at bottom, relations to times, just like all other temporary properties). She then extrapolates temporal multilocation and temporal relativization to personal multilocation and relativization, and, as a consequence, to spatial multilocation and relativization.

But I think anyone should reject such consequences out of hand. No selfsame subject can be both in the mountains and in the swampy place. And no one is both depressed and elevated, just at different persons. No

\footnotetext{
${ }^{23}$ Although this move seems to me rather natural for the BUNist to try I am not aware of any BUNists who actually make it. That is why the qualification 'hypothetical' may be appropriate.
}

Manuscrito - Rev. Int. Fil. Campinas, v. 40, n. 1, pp. 109-143, jan.-mar. 2017. 
amount of relativization will convince one otherwise. The intuition here is pretty clear: Lefty and Righty simply cannot be the same subject that has different experiences and is located at different places in a fundamentally relational way. ${ }^{24}$ Furthermore, any consideration compelling me to say that I am Righty should also lead me to say that I am Lefty. They have equal claims to represent $m y$ personal perspective. And since Lefty $\neq$ Righty the only way out of this quandary is to deny that there is a single selfsame me standing behind.

If this reply is compelling in the personal case it should, I contend, be equally compelling in the temporal case. In fact, the fission scenario already involves both dimensions at once. Many authors have noted that the pressure to deny that fission descendants of a split person are identical has the same source as the pressure to deny the identity between earlier and later stages of a persisting person, such as me-before-fission and Righty. Indeed, the lesson drawn by many from fission is that survival without strict identity is as good as it can get. And if it is not good enough in the fission case then it is no better in the ordinary case of day-to-day survival. ${ }^{25}$ Since the fission case strongly suggests that personal experiences (i.e. those of Lefty and Righty) cannot be at bottom relational (i.e., cannot be plausibly analyzed as the experiences of the selfsame me at Lefty and at Righty) the same holds true of the temporal experiences, thus favoring BUX over BUN.

I submit that the best BUNist response to this move is to go one step back and refuse to succumb to the temporal-personal analogy. The BUNist needs to reject the bait and deny that the way in which experiences and attendant beliefs are possessed by Lefty and Righty is relevantly similar to the way in which they are possessed by me-on-Tuesday and me-onWednesday. Perhaps the former are possessed simpliciter and "compartmentalized" in a way the latter are not. And perhaps temporal experiences (i.e. the experiences of a single person that are individuated by

\footnotetext{
24 This is a precise analog of the conclusion Skow draws from his spatial analogy in 2011: 378-379. See note 22. Skow now (2015: \$12.4) appears to be backing down from that conclusion, for reasons that are unclear to me at the moment.

${ }^{25}$ See, in particular, Parfit, op. cit.
}

Manuscrito - Rev. Int. Fil. Campinas, v. 40, n. 1, pp. 109-143, jan.-mar. 2017. 
times) are at bottom relational in a way personal experiences (i.e. the experiences of distinct persons) are not. Accordingly, the self may be "fragmented" or "scattered" across persons, but not across times. ${ }^{26}$ This would disqualify the intuitions grounded in the fission scenario from doing the same work in the temporal case, thus disarming the BUXist's present strategy.

The BUNist may add that this retort is dialectically appropriate, for the temporal-personal analogy as it is used in the above argument is far from being neutral in the broader context of the disagreement between endurantists and exdurantists. It may be very natural for the latter, but not for the former, to take the self to be divided across times in a way similar to that in which it is divided across its fission descendants. Consider again Lefty (one of my post-fusion descendants), Righty (my other descendant), and me (before the fission). The BUNist may agree that it is implausible to insist that I am elevated at Lefty and depressed at Righty, without compromising my strict self-identity. Me-at-Lefty is in the mountains and me-at-righty in the swampy place, so they cannot be identical. In general, no single enduring self can be wholly present and possess different properties "at two distinct persons" and, hence, at two distinct places. But the BUNist may insist that when it comes to time, the situation is not the same: time may interact with predication differently, making it very plausible to say that I am elevated on Tuesday and depressed on Wednesday, without compromising my strict identity over time. In sum, the BUNist may embrace the relational account of temporally distributed experiences but firmly reject the implausible relational analyses of personally and spatially distributed experiences.

I will now argue that this combination of views is inconsistent. The BUNist cannot have it both ways. The key to showing this is to "fuse" the temporal, personal, and spatial dimensions of self-location together in a manner that will block the BUNist from analyzing them differently. So far we have considered two types of cases: possession of different experiences by a single person at different times and possession of different

\footnotetext{
${ }^{26}$ Here and below I use 'self' as a neutral term not implying strict identity and 'person' as a more demanding term. Nothing turns on this choice of terminology; but we need a different term for each of these roles.
}

Manuscrito - Rev. Int. Fil. Campinas, v. 40, n. 1, pp. 109-143, jan.-mar. 2017. 
experiences by distinct persons (post-fission descendants of a single person) at different places. When we considered one of these cases we have tended to abstract from the other. But in the Block Universe, all three dimensions of self-location - the temporal, the personal, and the spatial are always present, even when some of them remain in the background. They can all be moved to the forefront and made to interact in a way revealing a hidden tension in the BUNist package of views. This will require further development of the scenario depicted in Fig. 5. I will do it in two steps.

First, suppose the fission happens on Tuesday. Next consider Lefty a bit later on Tuesday and Righty on Wednesday (Fig. 6). The former is still elevated, and the latter is still depressed.

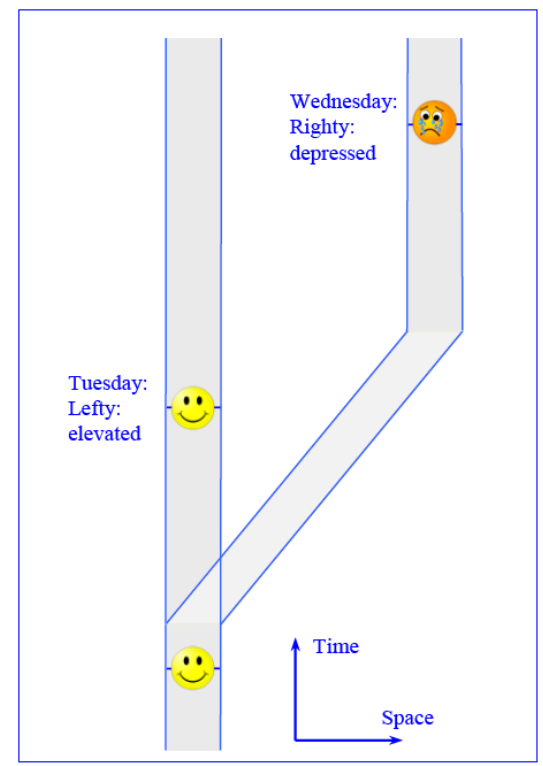

Fig. 6: Lefty on Tuesday and Righty on Wednesday.

The BUNist will reason about this case as before: the self may be scattered across persons, whenever and wherever they find themselves, but not across times. She may agree (looking at Lefty on Tuesday) that I am elevated simpliciter, and also that I am depressed simpliciter (now 
looking at Righty on Wednesday), and that in being depressed (and in finding myself on Wednesday and in the swampy place), I am not aware of being elevated (and of finding myself on Tuesday and in the mountains). She may, in other words, accept the picture of the self compartmentalized across the personal dimension (Fig. 7a).

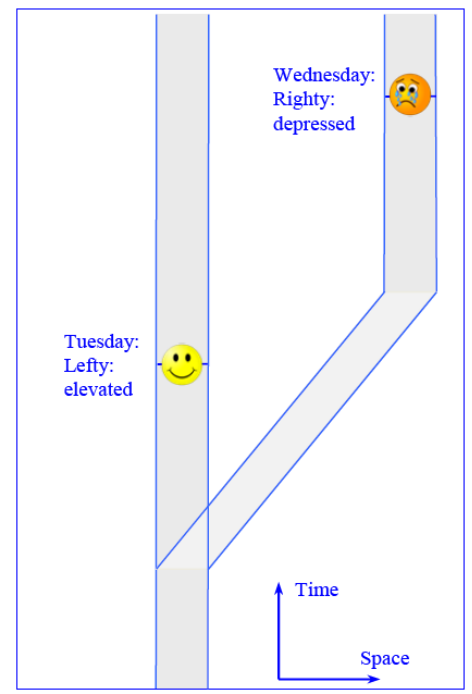

(a)

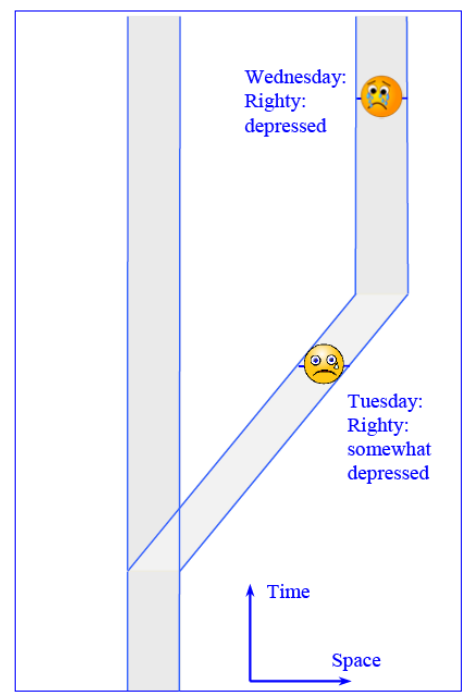

(b)

Fig. 7:

(a) Lefty on Tuesday $\neq$ Righty on Wednesday.

(b) Righty on Tuesday $=$ Righty on Wednesday.

But if the BUNist wants to treat the temporal dimension differently from the personal dimension she must refuse to say the same about the relation between Righty on Wednesday and Righty on Tuesday, shortly after the fission (Fig. 7b). That relation is simply identity, and the way in which Righty has his temporal experiences is at bottom relational. The BUNist may add that the difference between the two types of relation (non-identity in the first case, Fig. 7a; identity in the second, Fig. 7b) is clearly reflected in the presence of a unidirectional line (going from past to future) connecting Righty shortly after the fission on Tuesday to Righty 
on Wednesday (this line incorporates the relevant aspects of the spatiotemporal and causal continuity) and in the absence of such a connection between Lefty and Righty (despite the presence of the lines connecting both to me before the fission). So far so good. This completes the first step in the development of the scenario.

Now on to the second step. Suppose that fission is followed by a seamless fusion of Lefty with Righty on Wednesday, in the manner of Fig. 8.

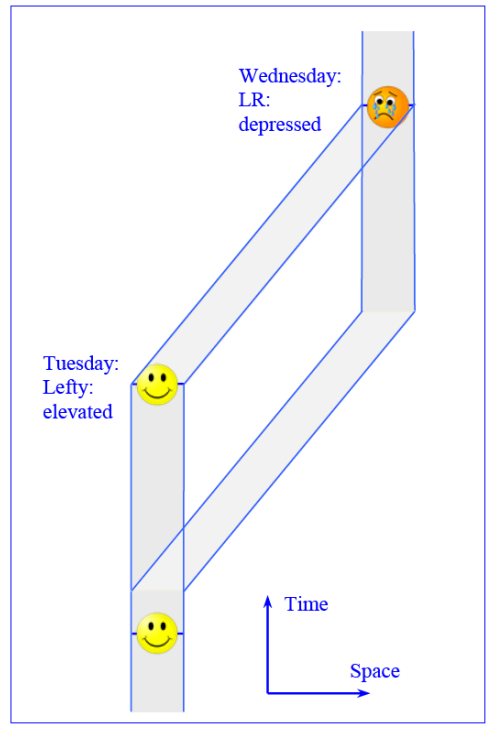

Fig. 8: Fission followed by fusion.

One way to think about such a scenario is to imagine that Lefty is slowly transported from the mountains to the swampy place, becoming increasingly depressed in the process, and then fused with Righty there. ${ }^{27}$ From then on, there is only one person in the scenario (call him LR), who is depressed. What is the relation between LR and Lefty on Tuesday? And what is the correct account of their experiences: the relational account based on strict identity, or the compartmentalized-self account which has

27 The idea of a seamless fusion of two or more persons was suggested and developed by Parfit (1971).

Manuscrito - Rev. Int. Fil. Campinas, v. 40, n. 1, pp. 109-143, jan.-mar. 2017. 
no room for it? From the BUNist point of view, the relation between LR and Lefty on Tuesday is essentially like the relation between LR and Righty on Tuesday shortly after the fission. Both relations involve two different moments of time but a single person; and each of them is now associated with a unidirectional continuity line. For the BUNist, then, (i) the experiences of LR on Wednesday and of Lefty on Tuesday must be at bottom relational (being in essence relations to these dates), and (ii) LR on Wednesday is strictly identical with Lefty on Tuesday.

But by the same token, (i) the experiences of LR on Wednesday and of Righty on Tuesday must be relational too, and (ii) LR on Wednesday is strictly identical with Righty on Tuesday. And the BUNist has already agreed that Lefty on Tuesday and Righty on Wednesday (who, by the assumption currently under consideration, is LR on Wednesday) cannot be the same person, and that their experiences are not at bottom relational, but are possessed simpliciter. After all, that would clearly be the case if the fusion did not happen (see Fig. 6). And being seamless, the fusion has no bearing on the plight of Righty. He would continue to be depressed regardless. So LR on Wednesday and Lefty on Tuesday are and are not strictly identical, and their experiences are and are not at bottom relational.

This clearly unacceptable result stems from the peculiar way in which the fusion leg of the scenario brings together the temporal and the personal dimensions of self-location, thus enforcing two different ways of having temporal experiences - relative to times, and simpliciter, the first of which requires strict identity across time and the second rules it out. Let us reflect on this result more carefully, by walking again through the two stages of the scenario.

The branching aspect of the initial fission suggests the familiar fragmentation of the self in a manner typically described in the literature, leading to the conclusion that Lefty on Tuesday and Righty on Tuesday are not identical and possess their incompatible experiences simpliciter. Since LR, by assumption, is strictly identical to Righty on Tuesday, the same conclusion must be drawn about Lefty on Tuesday and LR: they cannot be identical. This conclusion can be reinforced by imagining that the subsequent fusion does not occur (Fig. 6 and 9a).

Now focus on the fusion stage, which comes with a new continuity line connecting Lefty on Tuesday and LR (Fig. 9b). By parity of reasoning

Manuscrito - Rev. Int. Fil. Campinas, v. 40, n. 1, pp. 109-143, jan.-mar. 2017. 
(and in accordance with the package of views the BUNist wants to have), this line enforces strict identity of Lefty on Tuesday and LR. (It also enforces possession of their incompatible experiences, not simpliciter, but only relative to times.) This conclusion can be reinforced by temporarily eliminating Rightly from the picture (remember that the fusion is expected to be seamless).

Thus, when the BUNist reflects on the first stage of the scenario (and abstracts from the second) she is naturally driven by the characteristic "non-identity intuitions" amply described in the literature on fission (see Fig. 9a). But when the BUNist turns to the fusion leg of the scenario (while abstracting from its first stage; see Fig. 9b) she is pulled by the characteristic "identity intuitions," for the situation now involves mundane change over time, and the BUNist has a familiar relational story about it. But at the end of the day, both sorts of intuitions apply to the same items, Lefty on Tuesday and LR, explicitly juxtaposed in the personal as well as the temporal dimension.

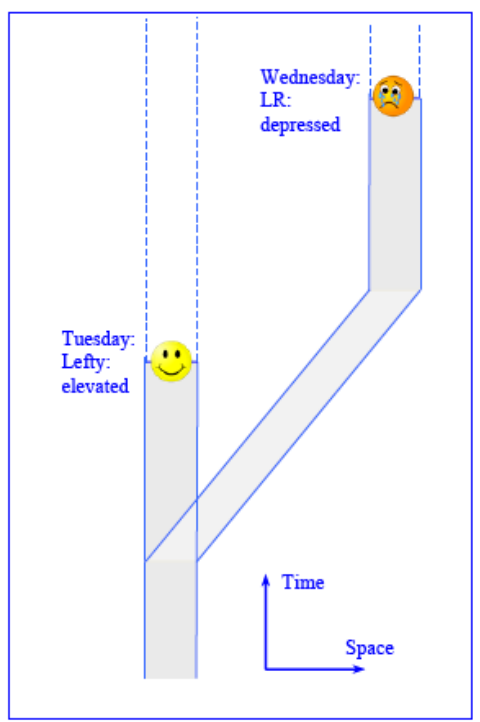

(a)

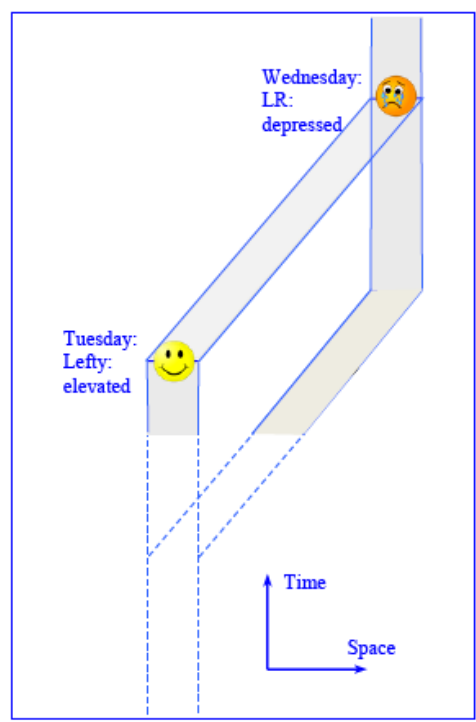

(b)

Fig. 9: (a) Lefty on Tuesday $\neq$ LR. (b) Lefty on Tuesday $=L R$. 
As noted earlier, both dimensions are already present in the more standard fission scenarios: Lefty and Rightly are separate persons, but they need not be considered at the same time. Most of the literature on fission, however, abstracts from the temporal dimension of the situation and focuses almost exclusively on its personal dimension. Of course, this is done for a good reason: the personal dimension takes the center stage because of its paramount importance and the controversy associated with it. Discussions of temporal experience, on the other hand, very rarely mention fission; again, for a good reason. They tend to abstract from the personal dimension altogether and focus on the all-important temporal dimension of experience.

Fusion helps to bring both dimensions together by producing LR, a single person, who is related to Lefty on Tuesday "twice over": as a later stage of him, but also as a previous fission counterpart. According to BUN, the former relation requires identity, but the second prohibits it. But of course, it cannot be both ways.

BUX, in contrast, offers a consistent view, by refusing to consider any across-time relation as identity in the first place. LR is simply non-identical with Lefty on Tuesday. LR is also non-identical with Righty on Tuesday. And he has his temporal properties simpliciter, not relative to times.

I see only two ways for the BUNist to avoid the above-noted inconsistency in his preferred package of views: (i) become a BUXist, agree that all experiences - personal, as well as temporal - are had simpliciter, and embrace the picture of the self compartmentalized in both these dimensions; or (ii) take a very pessimistic stand on fusion, as well as fission.

What does (ii) involve? The BUNist needs to claim that, when fission happens, the original person goes out of existence and two new persons, Lefty and Righty, are created. In the same vein, the BUNist must insist that when the fusion happens subsequently, Lefty and Righty go out of existence, and a numerically distinct new person, LR, comes into being. This eliminates the pressure to treat LR as a later stage of Lefty on Tuesday (for they are now numerically distinct) and obviates the need to consider them "twice over" (as temporal stages of a single person, and as two distinct persons). But treating Lefty on Tuesday and LR as numerically different does not preclude the relational analyses of their corresponding

Manuscrito - Rev. Int. Fil. Campinas, v. 40, n. 1, pp. 109-143, jan.-mar. 2017. 
temporal experiences. More generally, there are four numerically distinct persons in the entire scenario: me before fission, Lefty, Rightly, and LR; and each of them has temporal experiences relationally, thus saving BUN.

Being so pessimistic about fusion may initially seem just as natural for the BUNist as being antecedently pessimistic about fission. If the forwardlooking branching destroys the original person then why should the backward-looking branching promise a better result? But in one sense, the fusion part of the scenario is different. Assuming it to be seamless, fusion does not involve any intrinsic change to any existing person - very unlike fission. Instead, it simply adds an external counterpart to an existing person, Rightly. Insisting that this external addition results in Righty going out of existence is hardly acceptable. The BUNist may be motivated to deny the continuing existence of the self after fission precisely on the grounds that no entity can survive literal splitting in two. But this motivation does not apply to fusion, where something external is added to a person already assumed to be in existence.

But only the fusion part of the scenario is essentially involved in generating the above-noted inconsistency in the BUNist preferred combination of views. One could imagine that fission did not happen at all, and the scenario starts with Lefty and Righty on Tuesday being fused into LR on Wednesday. The BUNist wishing to pursue the pessimistic option (ii) will thus have to say that, although Righty's fusion with Lefty is seamless, Righty and Lefty go out of existence in the process, and a new person, LR, is created. I think this raises the cost of the resulting position rather significantly, and (i) is a much better option. ${ }^{28}$ But I must leave the matter here.

${ }^{28}$ For one thing, LR will say that he is Righty. And if Righty was involved in a mental operation of adding 37 and 29 just before the fusion LR will say "66" immediately thereafter, without taking any note of the fusion. LR will also say that he is Lefty. For another thing, the extended argument of this paper could be restated in terms of a slightly more down-to-earth scenario involving cutting (easy) and reconnecting (difficult) corpus callosum, thereby creating and then merging back together two relatively independent "streams of consciousness." For a very useful and detailed, if opinionated, survey of the data pertaining to the split-brain syndrome, see Bayne 2010: Ch. 9.

Manuscrito - Rev. Int. Fil. Campinas, v. 40, n. 1, pp. 109-143, jan.-mar. 2017. 


\section{Concluding Remarks}

This whole discussion has revolved around persons and experiences, pushing the broader metaphysical issues to the periphery. It may be helpful to briefly revisit some of them and take stock of the unfinished business.

First, the argument is intended to raise the cost of endurance in the Block Universe. It does so by directly raising the cost of BUN for persons, and only indirectly for other objects. Nothing in the argument poses a problem for the view that rocks, computers and cells persist over time by enduring and have their temporary properties in a fundamentally relational way, rather than simpliciter. The argument gets its bite from the need to explain away a false belief that each of us is viewing the Block Universe exclusively from a particular temporal perspective (rather than from a different such perspective). Rocks, sticks, computers and cells simply do not have any perspectives for us to worry about. The selfsame stick can be bent at one time in virtue of standing in the bent-at relation to it, and straight at another time in virtue of standing in the straight-at relation to it; and that can be the whole story about it. ${ }^{29}$ In this respect, the puzzle of temporal experience is not just the problem of temporary intrinsics in disguise, despite the very real and important similarity between them. ${ }^{30}$

To be sure, the view that atoms, molecules and cells persist by enduring but persons composed of them by exduring would be difficult to defend.

Second, the discussion has been focused solely on experiencing the present and has left other intriguing and difficult aspects of temporal experience aside, such as the perception of the dynamical passage of time. But it is good to take big problems one at a time.

Other issues put aside have to do with the duration of the temporal experiences, the "specious present," other related notions, and the need

\footnotetext{
29 And if (implausibly) the stick can be wholly present at two different places at once, it can be bent at one place in virtue of standing in the bent-at relation to that place, and straight at another in a similar way; and that can be the whole story about it.

${ }^{30}$ For more on this similarity, see Balashov 2005: $\int 4.2$; Skow 2011: $\iint 5-7$, and notes 22 and 26; and Skow, 2015, Ch. 12, notes 4 and 20.
}

Manuscrito - Rev. Int. Fil. Campinas, v. 40, n. 1, pp. 109-143, jan.-mar. 2017. 
to square all that with the idealization of instantaneous stages assumed throughout. $^{31}$

Finally, I have expressed concerns over the standard way of developing BUX which starts by drawing close modal-temporal analogies, and pushed for an alternative way inspired by personal-temporal analogies leading to the picture of a thoroughly compartmentalized self. At this point it is just a picture, and a long way from a theory of the self. Furthermore, the picture must eventually be generalized to all objects, and much more needs to be said about its consequences for the statements of identity, predication, and related matters. I hope to turn to these matters in the future. $^{32}$

\section{References}

Balashov, Y. "Times of Our Lives: Negotiating the Presence of Experience," American Philosophical Quarterly 42: 295-309, 2005.

"Experiencing the Present," Epistemology and Philosophy of Science 44: 61-73, 2015.

Bayne, T. The Unity of Consciousness. Oxford: Oxford University Press, 2010.

\footnotetext{
31 See, in this connection, Sider 2001: 197-198, Hawley 2001: 50f, and Balashov 2005: $\int 5$.

${ }^{32}$ One crucial and pressing question is: if the relation between me and my temporal stages is not identity, what is it? Here are some options. (i) Despite many considerations to the contrary, this relation is identity: I am my stages, and they are me. (ii) This relation is indeterminate identity: I am indeterminately my Tuesday stage, and I am indeterminately my Wednesday stage; my Tuesday stage is neither determinately nor indeterminately my Wednesday stage; however, I am determinately one of my stages. (iii) There is simply no "me" in this picture, there are only "my" stages. At this point I am inclined to reject (i) and undecided between (ii) and (iii). I am firmly convinced, however, that the way in which the self is "scattered" across time (if it is) and the way in which it is "scattered" across fission branches (if it is) are fundamentally the same.
}

Manuscrito - Rev. Int. Fil. Campinas, v. 40, n. 1, pp. 109-143, jan.-mar. 2017. 
Grünbaum, A. "The Exclusion of Becoming from the Physical World," in M. Čapek, ed., The Concepts of Space and Time (Dordrecht: Reidel), pp. 471-500, 1976.

Haslanger, S. "Persistence Through Time," in M.J. Loux and D.W. Zimmerman (eds.), The Oxford Handbook of Metaphysics (Oxford: Oxford University Press), pp. 315-54, 2003.

Hawley, K. How Things Persist. Oxford: Clarendon Press, 2001.

Hoy, R. "Becoming and Persons," Philosophical Studies 34: 269-280, 1978.

Lewis, D. "Attitudes De Dicto and De Se," Philosophical Review 88: 513-543, 1979.

"Survival and Identity," in D. Lewis, Philosophical Papers (Oxford: Oxford University Press), Vol. 1, pp. 55-77, 1983. On the Plurality of Worlds. (Oxford: Basil Blackwell), 1986.

Mellor, D.H. (1998), Real Time II. London and New York: Routledge, 1998.

Merricks, T. "On the Incompatibility of Enduring and Perduring Entities," Mind 104: 523-531, 1995.

Oaklander, L. N. "On the Experience of Tenseless Time," in N.L. Oaklander and Q. Smith, eds., The New Theory of Time (New Haven and London: Yale University Press), pp. 344-350, 1994.

Parfit, D. "Personal Identity," Philosophical Review 80: 3-27, 1971. Reasons and Persons. Oxford: Clarendon Press, 1984.

"Divided Minds and the Nature of Persons," in P. van Inwagen and D. Zimmerman, eds., Metaphysics: The Big Questions, 2nd ed. (Oxford: Blackwell Publishing), pp. 361-362, 2008.

Perry, J. “The Problem of the Essential Indexical," Nồs 13: 3-21, 1979.

Sider, T. Four-Dimensionalism: An Ontology of Persistence and Time. Oxford: Clarendon Press, 2001.

Manuscrito - Rev. Int. Fil. Campinas, v. 40, n. 1, pp. 109-143, jan.-mar. 2017. 
Skow, B. "Experience and the Passage of Time," Philosophical Perspectives 25: 359-387, 2011.

Objective Becoming. Oxford: Oxford University Press, 2015.

Torre, S. "Tense, Timely Action and Self-Ascription," Philosophy and Phenomenological Research 80: 112-132, 2010.

Manuscrito - Rev. Int. Fil. Campinas, v. 40, n. 1, pp. 109-143, jan.-mar. 2017. 